\title{
Transcriptome and metabolome analysis to reveal major genes of saikosaponin biosynthesis in Bupleurum chinense
}

Yilian He ${ }^{1 \dagger}$, Hua Chen ${ }^{1 \dagger}$, Jun Zhao ${ }^{1}$, Yuxia Yang ${ }^{2}$, Bin Yang ${ }^{1}$, Liang Feng ${ }^{1}$, Yiguan Zhang ${ }^{3}$, Ping Wei ${ }^{3}$, Dabin Hou', Junning Zhao ${ }^{3^{*}}$ and Ma Yu, $\mathrm{H}^{1,4^{*}}$

\begin{abstract}
Background: Bupleurum chinense DC. is a widely used traditional Chinese medicinal plant. Saikosaponins are the major bioactive constituents of $B$. chinense, but relatively little is known about saikosaponin biosynthesis. In the present study, we performed an integrated analysis of metabolic composition and the expressed genes involved in saikosaponin biosynthetic pathways among four organs (the root, flower, stem, and leaf) of B. chinense to discover the genes related to the saikosaponin biosynthetic pathway.

Results: Transcript and metabolite profiles were generated through high-throughput RNA-sequencing (RNA-seq) data analysis and liquid chromatography tandem mass spectrometry, respectively. Evaluation of saikosaponin contents and transcriptional changes showed 152 strong correlations $(P<0.05)$ over 3 compounds and 77 unigenes. These unigenes belonged to eight gene families: the acetoacetyl CoA transferase (AACT) (6), HMG-CoA synthase (HMGS) (2), HMG-CoA reductase (HMGR) (2), mevalonate diphosphate decarboxylase (MVD) (1), 1-deoxy-Dxylulose-5-phosphate synthase (DXS) (3), farnesyl diphosphate synthase (FPPS) (11), $\beta$-amyrin synthase ( $\beta$-AS) (13) and cytochrome P450 enzymes (P450s) (39) families.

Conclusions: Our results investigated the diversity of the saikosaponin triterpene biosynthetic pathway in the roots, stems, leaves and flowers of $B$. chinese by integrated transcriptomic and metabolomic analysis, implying that manipulation of P450s genes such as Bc95697 and Bc35434 might improve saikosaponin biosynthesis. This is a good candidate for the genetic improvement of this important medicinal plant.
\end{abstract}

Keywords: Bupleurum chinense DC. Transcriptome, Metabolome, Saikosaponin biosynthetic pathway

\footnotetext{
*Correspondence: zarmy@189.cn; mayu0073@swust.edu.cn

${ }^{\dagger}$ Yilian He and Hua Chen contributed equally to this work.

${ }^{3}$ Sichuan Institute for Translational Chinese Medicine, Chengdu 610041,

China

${ }^{1}$ School of life science and engineering, Southwest University of Science and Technology, 59 Qinglong Road, Mianyang 621010, Sichuan, China Full list of author information is available at the end of the article
}

(c) The Author(s). 2021 Open Access This article is licensed under a Creative Commons Attribution 4.0 International License, which permits use, sharing, adaptation, distribution and reproduction in any medium or format, as long as you give appropriate credit to the original author(s) and the source, provide a link to the Creative Commons licence, and indicate if changes were made. The images or other third party material in this article are included in the article's Creative Commons licence, unless indicated otherwise in a credit line to the material. If material is not included in the article's Creative Commons licence and your intended use is not permitted by statutory regulation or exceeds the permitted use, you will need to obtain permission directly from the copyright holder. To view a copy of this licence, visit http://creativecommons.org/licenses/by/4.0/ The Creative Commons Public Domain Dedication waiver (http://creativecommons.org/publicdomain/zero/1.0/) applies to the data made available in this article, unless otherwise stated in a credit line to the data. 


\section{Background}

Plants of the genus Bupleurum are major components of Oriental folk medicine in China, Japan and Korea, and have been officially listed in the Chinese and Japanese Pharmacopoeias [1]. These species are used either alone or in combination with other ingredients for treatment of the common cold, inflammatory disorders, hepatitis and fever [2]. Up to now, phytochemical studies have shown that Bupleurum contains abundant natural compounds, such as phenolics, lignans, terpenoids (triterpenoids and sterols), mono- and sesquiterpenes (essential oils) and polyacetylenes [3]. Among them, saikosaponins (SSs) are considered as the principal bioactive components [4]. SSs generally constitute the main class of secondary metabolites in the genus Bupleurum, accounting for up to $7 \%$ of the total dry weight of the roots [2].

In recent years, more than 120 glycosylated oleananetype and ursane-type SSs have been isolated from Bupleurum species [5]. A representative biosynthetic pathway for triterpenoid saponins in Bupleurum was described by Zheng et al., [6]: isopentenyl diphosphate (IPP) and dimethylallyl diphosphate (DMAPP) are produced by the mevalonate (MVA) pathway and the methylerythritol phosphate (MEP) pathway. IPP and DMAPP synthesize 2,3-oxidosqualene, which is catalyzed by geranyl pyrophosphate synthetase (GPS), FPPS, squalene synthetase (SS) and squalene epoxidase (SE). 2,3-Oxidosqualene is cyclized by $\beta$-AS to produce $\beta$-amyrin. Finally, $\beta$-amyrin is oxidatively modified and glycosylated to produce various types of SSs by P450s and uridine diphosphate glycosyltransferases (UGTs) (Fig. 1). In our previous studies, candidate genes responsible for saikosaponin biosynthesis were identified in $B$. chinense on the basis of the coordinated upregulation of their expression with $\beta$-AS in methyl jasmonate-treated adventitious roots, especially P450s and UGTs [7]. However, the regulatory mechanism for the saikosaponin biosynthesis pathway is still unknown.

Integrated omics analysis of the transcriptome and metabolome has become an effective tool to better understand biosynthetic pathways [8]. This technique enables identification of novel functional genes and characterization of the regulatory mechanisms and key factors of biosynthetic pathways. The regulatory genes in the flavanol [9], anthocyanin [10] and ginsenoside biosynthesis pathways [11] have been explored using integrated analysis, and potential target genes have been identified.

In this study, we used $B$. chinense to 1) explore the regulatory networks of the saikosaponin synthesis pathway in this species at the level of transcriptome and metabolome, 2) identify the differential expression of saikosaponin metabolites and their regulatory genes among the roots, stems, leaves and flowers at the adult stage, and 3) distinguish the key factors associated with saikosaponin biosynthesis.

\section{Results}

Metabolic profiling among the root, stem, leaf and flower A total of 600 metabolites were identified in the samples, and all of them accumulated differentially in the four organs (Additional file 1: Table S1). To compare the compositions of metabolites involved in saikosaponin synthesis in the four organs, datasets obtained from high-performance liquid chromatography-tandem mass spectrometry (HPLC-MS/MS) in the ESI+ and ESImodes were subjected to Principal component analysis (PCA). The results showed that the four organs were separated in the PC1 $\times$ PC2 score plots. Indeed, PC1 and PC2 in ESI+ and ESI- modes (39.17 and 20.90\%,

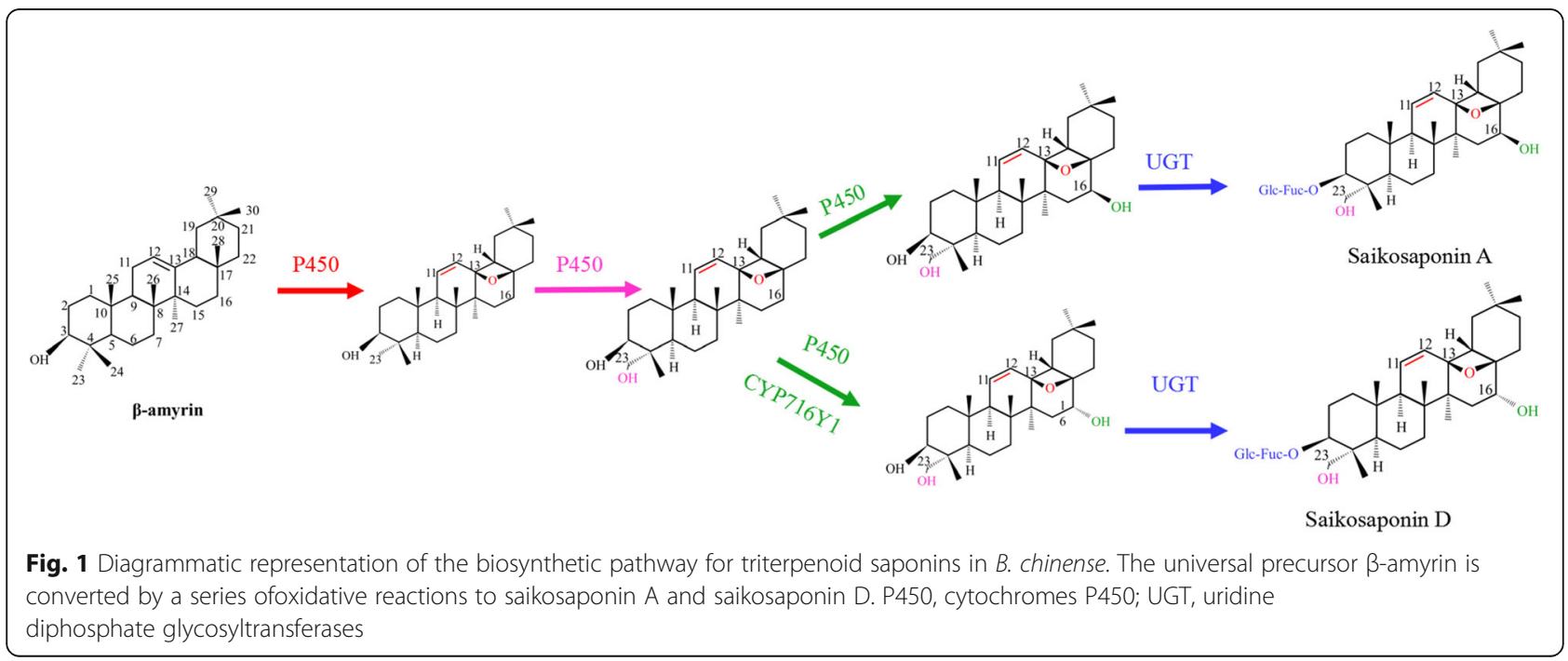


respectively) were clearly separated among the roots, stems, leaves and flowers (Fig. 2A). This grouping indicated that the accumulation pattern of metabolites varied among organs. Selection of the variables responsible for the differences was performed through statistical analysis as described in the Materials and Methods. A total of 76,81 , and 79 mass ions were selected between roots and stems, between roots and leaves, and between roots and flowers in the ESI+ and $\mathrm{ESI}^{-}$modes, respectively (Fig. 2B).

We detected four triterpenoids among 10 terpenoids, including saikosaponin A (SSa), saikosaponin B (SSb), saikosaponin D (SSd) and momordin Ic. In addition, two sesquiterpene lactones (artemisinin and atractylenolide I), two monoterpenoids (rehmannioside $\mathrm{A}$ and albiflorin), one diterpenoid (kaurenoic acid) and one triterpenic acid (betulonic acid) were detected. The relative abundance of triterpenoids in organs was much higher than other terpenoids, except in the stem. The relative abundance of sesquiterpene lactones in the stem was higher than that in other three organs. SSb and SSd were the most abundant components of the root extracts (Table 1). The root was the most active organ regarding to SSb and SSd accumulation, while flower was the most active organ for SSa.

HPLC analysis revealed that the relative abundance of SSs differed among the four organs (Additional file 1: Table S2). The abundance of the SSd and SSa was only found in root.
Transcriptome analysis of the root, stem, leaf and flower Through RNA-Seq, a total of 12 samples in the four organs independently yielded numbers of raw RNA-Seq reads ranging from 40,761,460 to 77,506,190 (Table 2). After stringent quality checks and removal of adaptor sequences, clean paired-end reads ranging from 40,243,798 to $76,382,598$ were acquired. Then, the expression profiling of genes involved in the saikosaponin biosynthetic pathway was subsequently performed. Between 69.84 and $81.54 \%$ of the clean reads were mapped. Finally, a total of 93,486 unigenes were assembled (Additional file 1: Table S3).

To investigate the functions of the unigene set transcripts, a sequence similarity search was conducted using protein databases including the non-redundant (nr), Swiss-Prot, and Pfam databases using BLASTx (cutoff Evalue $\left.<1 \mathrm{e}^{-10}\right)$. The results indicated that 89,488 (95.72\%), 78,155 (83.60\%), and 61,248 (65.52\%) transcripts shared significant similarity with protein sequences in the nr, Swiss-Prot, and Pfam databases, respectively. In addition, we used BLAST to search the nucleotide sequence databases, including the nucleotide (nt) database, and 81,779 (87.48\%) transcripts were also significantly similar to nucleotide sequences.

Gene Ontology (GO) analysis was performed to classify the functions of the assembled transcripts. Based on the GO analysis, a total of 61,248 (65.52\%) unigenes were assigned to $2137 \mathrm{GO}$ terms. The top GO terms of all the transcripts were categorized into 54 functional

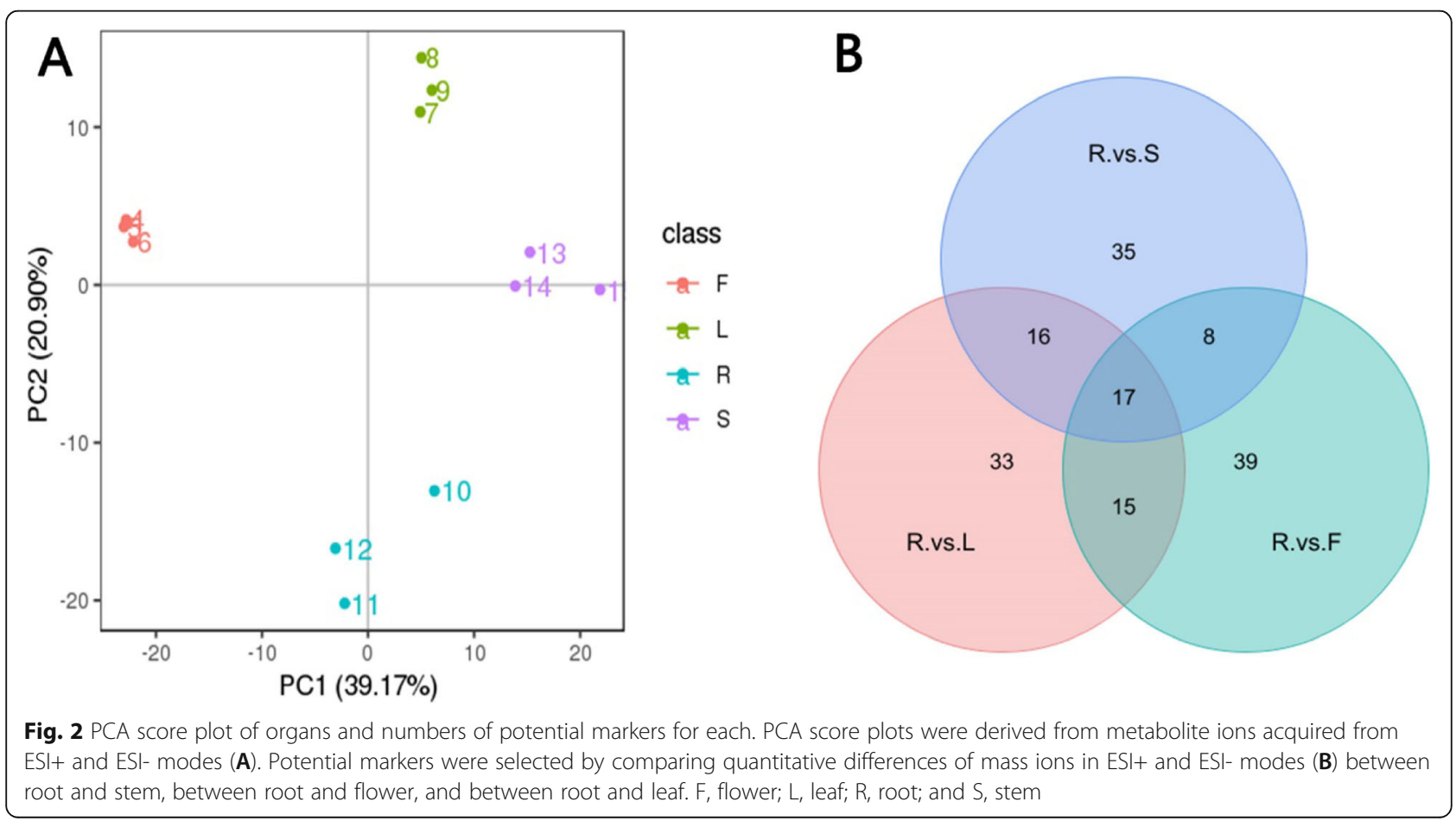




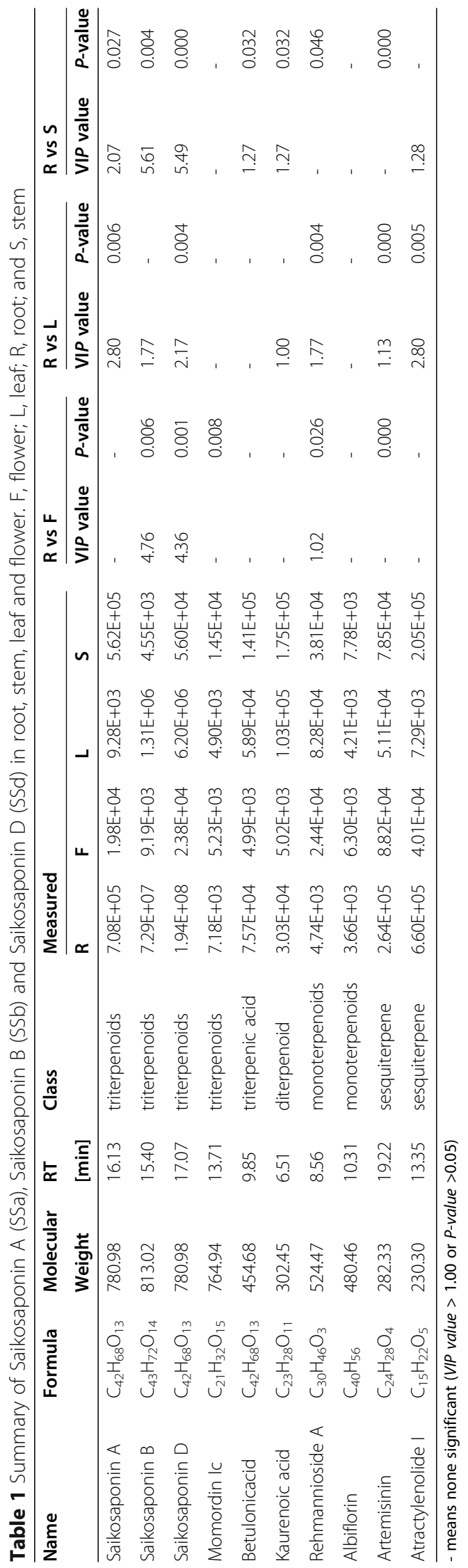


Table 2 Summary of transcriptome data generated in root, stem, leaf, flower

\begin{tabular}{|c|c|c|c|c|c|c|}
\hline Sample name & Raw reads & Clean reads & Clean bases(G) & Error rate(\%) & Q20(\%) & Total mapped \\
\hline root1 & $61,923,840$ & $61,278,296$ & 9.19 & 0.02 & 98.22 & $49347822(80.53 \%)$ \\
\hline root2 & $59,085,774$ & $56,835,612$ & 8.53 & 0.03 & 97.71 & $46164456(81.22 \%)$ \\
\hline root3 & $50,949,232$ & $50,224,936$ & 7.53 & 0.02 & 98.20 & $39732948(79.11 \%)$ \\
\hline stem1 & $52,918,430$ & $52,169,174$ & 7.83 & 0.02 & 98.19 & $41240630(79.05 \%)$ \\
\hline stem2 & $49,945,800$ & $49,481,408$ & 7.42 & 0.02 & 98.36 & $40467226(81.78 \%)$ \\
\hline stem3 & $49,924,560$ & $49,120,966$ & 7.37 & 0.02 & 98.31 & $39130028(79.66 \%)$ \\
\hline leaf1 & $40,761,460$ & $40,243,798$ & 6.04 & 0.02 & 98.34 & $32651514(81.13 \%)$ \\
\hline leaf2 & $53,021,426$ & $52,225,618$ & 7.83 & 0.02 & 98.13 & $42585984(81.54 \%)$ \\
\hline leaf3 & $52,257,396$ & $51,626,318$ & 7.74 & 0.02 & 98.14 & 41497244(80.38\%) \\
\hline flower1 & $77,506,190$ & $76,382,598$ & 11.46 & 0.02 & 98.15 & $53983590(70.68 \%)$ \\
\hline flower2 & $53,204,672$ & $52,616,858$ & 7.89 & 0.03 & 98.10 & $37283804(70.86 \%)$ \\
\hline flower3 & $56,907,780$ & $56,118,732$ & 8.42 & 0.02 & 98.09 & $39192864(69.84 \%)$ \\
\hline total & $658,406,560$ & $648,324,314$ & & & & \\
\hline
\end{tabular}

groups in three main categories, namely, the biological process, molecular function, and cellular component categories. As shown in Fig. 3, in the biological process category, the most highly encoded proteins were those associated with the metabolic process term (GO:0008152, 29,115 unigenes, $47.54 \%$ ), followed by those associated with the cellular process (GO:0009987, 43.00\%) and single-organism process (GO:0044699, 28.21\%) terms, indicating that the plants were undergoing extensive metabolic activity. In the molecular function category, binding (GO:0005488, 38,826 unigenes, 61.76\%), catalytic activity
(GO:0003824, 48.41\%) and transporter activity (GO: $0005215,4.97 \%)$ were well-represented terms. In the cellular component category, the genes were mostly enriched for the cell (GO:0005623, 11,179 unigenes, 18.25\%) and cell part (GO:0043226, 18.25\%) terms.

\section{Comparison of saikosaponin synthesis pathways among the root, stem, leaf, and flower}

In order to better understand saikosaponin synthesis pathways, all the unigenes were analyzed using the Kyoto Encyclopedia of Genes and Genomes (KEGG)

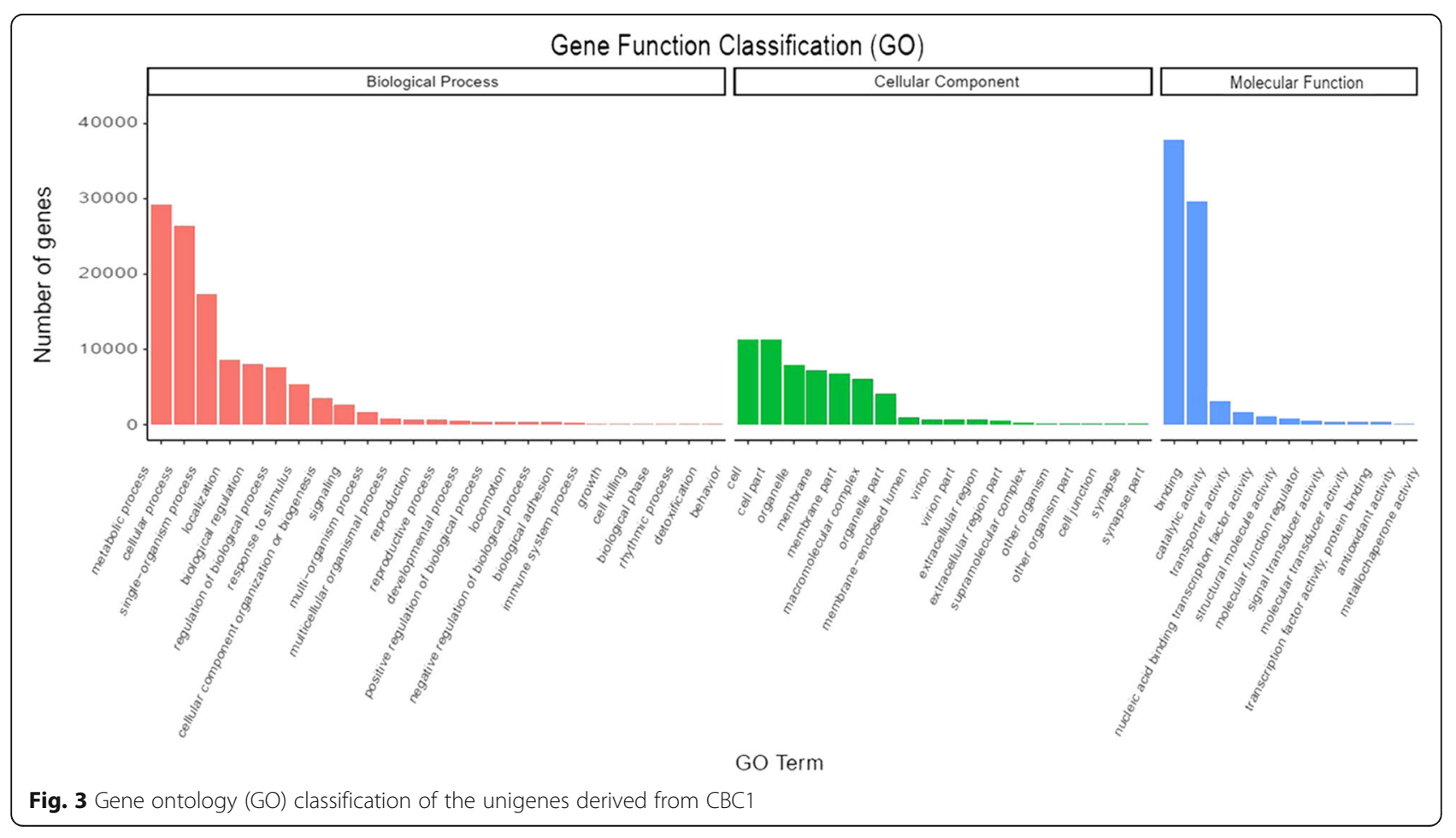


pathway database. KEGG database contains records of triterpenoid pathways and molecular interactions in organisms cells. In total, 241 and 114 unigenes were annotated in the terpenoid backbone biosynthesis (map 00900) and sesquiterpenoid and triterpenoid biosynthesis (map 00909) pathways, respectively.

To seek for genes involved in the differential catabolism of saikosaponin in four organs, the abundance and expression patterns of all gene transcripts were analyzed based on their fragments per kilobase of exon per million fragments (FPKM) transcriptome data. Differentially expressed genes (DEGs) in the MVA pathway were found in transcriptome through individual assembling data and were divided into five families: the AACT family (7 unigenes), the HMGS family (5 unigenes), the HMGR family (8 unigenes), the MVK family (2 unigenes), and the MVD family (3 unigenes). For the DEGs involved in the MEP pathway, only one gene family, the DXS family (8 unigenes), was identified. The triterpene biosynthesis-related DEGs included SS (11 unigenes), $\beta$-AS (24 unigenes), P450s (49 unigenes) and UGTs (14 unigenes), which catalyze the conversion of oleanane-type SSs (Fig. 4; Additional file 1: Table S4).

\section{Correlations between unigenes and metabolites}

In order to understand the regulatory network for the differential distribution of SSs among roots, stems, leaves and flowers, the relative abundance of SSa, SSb and SSd was determined in the metabolite study, and Pearson correlation analysis was performed with 131 DEGs that were categorized into saikosaponin synthetic pathways. The correlation analysis results showed that 96 unigenes had strong correlation coefficient values $(P<0.05)$ with 3 metabolites. Based on the results, networks of interactions among the metabolites and unigenes were organized for the roots, stems, leaves and flowers.

The relative abundance levels of SSb and SSd were greater in roots than in other organs and were highly correlated with the unigenes in the AACT, FPPS, HMGR, $\beta$-AS, MVD and P450s clusters (Fig. 5). On the other hand, SSa was more predominant in flowers than in other organs and was shown to be highly connected with the unigenes in the P450s cluster.

\section{Real-time polymerase chain reaction (PCR) validation}

Real-time PCR was used to experimentally verify the relative expression of putative unigenes involved in the biosynthesis of saikosaponin of B. chinense. Fifteen unigenes including the genes putatively encoding enzymes involved in the gene families of AACT, FPPS, HMGR, HMGS, MVD, P450 and $\beta$-AS were tested among different tissues of the plant with three replicates (Fig. 6). The real-time PCR analysis suggested that 11 of fifteen unigenes displayed a higher transcript pattern in root, as compared with other organs; whereas only four genes, Bc14211 (encoding AACT), Bc37389 (encoding DXS), $B c 47895$ (encoding FPPS) and Bc49808 (encoding $\beta$-AS) were noticed a higher level in flowers. High expression level of these unigenes in the roots of the plant indicated that SSs are highly produced in the roots. These results were in keep with RNA-seq information analysis.

\section{Subcellular localization of P450s in N. benthamiana cells} Bc95697 and Bc35434 are both genes encoding P450s. In order to understand their function, the subcellular localization was analyzed. Confocal microscope observation showed that controls expressing a 35S-yellow fluorescent protein (YFP) construct showed fluorescence labeling in endoplasmic reticulum and nucleoplasm. The fluorescence signal of Bc95697-YFP and Bc35434-YFP fusion protein in tobacco epidermal cells are obviously located in the endoplasmic reticulum (Fig. 7), indicating that Bc95697 and Bc35434 are active proteins in the endoplasmic reticulum.

\section{The high content of two saikosaponins in four genetic types accompanied with the tissue-specific expression of $B c 95697$ and $B c 35434$ genes}

To explore the roles of $B c 95697$ and Bc35434. Two genes from $\mathrm{CBC} 1, \mathrm{CBC} 3, \mathrm{FS}-1$ and $\mathrm{RX}-1$ were analyzed by real-time PCR analysis. We found the expression of Bc95697 and Bc35434 at different genetic type of Bupleurum has tissue-specific expression, and the expression in root was obviously higher than in other organs (Fig. 8). In line with the higher expression of the genes, liquid chromatography-mass spectrometry (LCMS) analysis suggested that the abundance of SSa and SSd showed a significant higher $(P<0.01)$ in root.

\section{Discussion}

We characterized 10 terpenoids in four organs, four of which were triterpenes. Oleanane-type SSs (SSa, SSd and $\mathrm{SSb}$ ) formed the major group of triterpenes in the root, leaf, and flower of the plant, which is consistent with a previous report that SSa and SSd are the most abundant triterpene saponins found in Bupleurum species [12]. SSs with variations in component abundance and total saponin content among different plants are easily affected by the environment [13]. Previous methods such as thin-layer chromatography (TLC) and HPLC, were limited to detect a few active components with relative high abundance, such as SSa, SSc and SSd [13]. The continual refinement and increasing scope and scale of plant metabolite profiling methods have led to the evolution of modern plant metabolomics, which has matured as a valuable tool for advancing our understanding of plant biology and physiology [14]. This detection technique has been successfully applied for the 


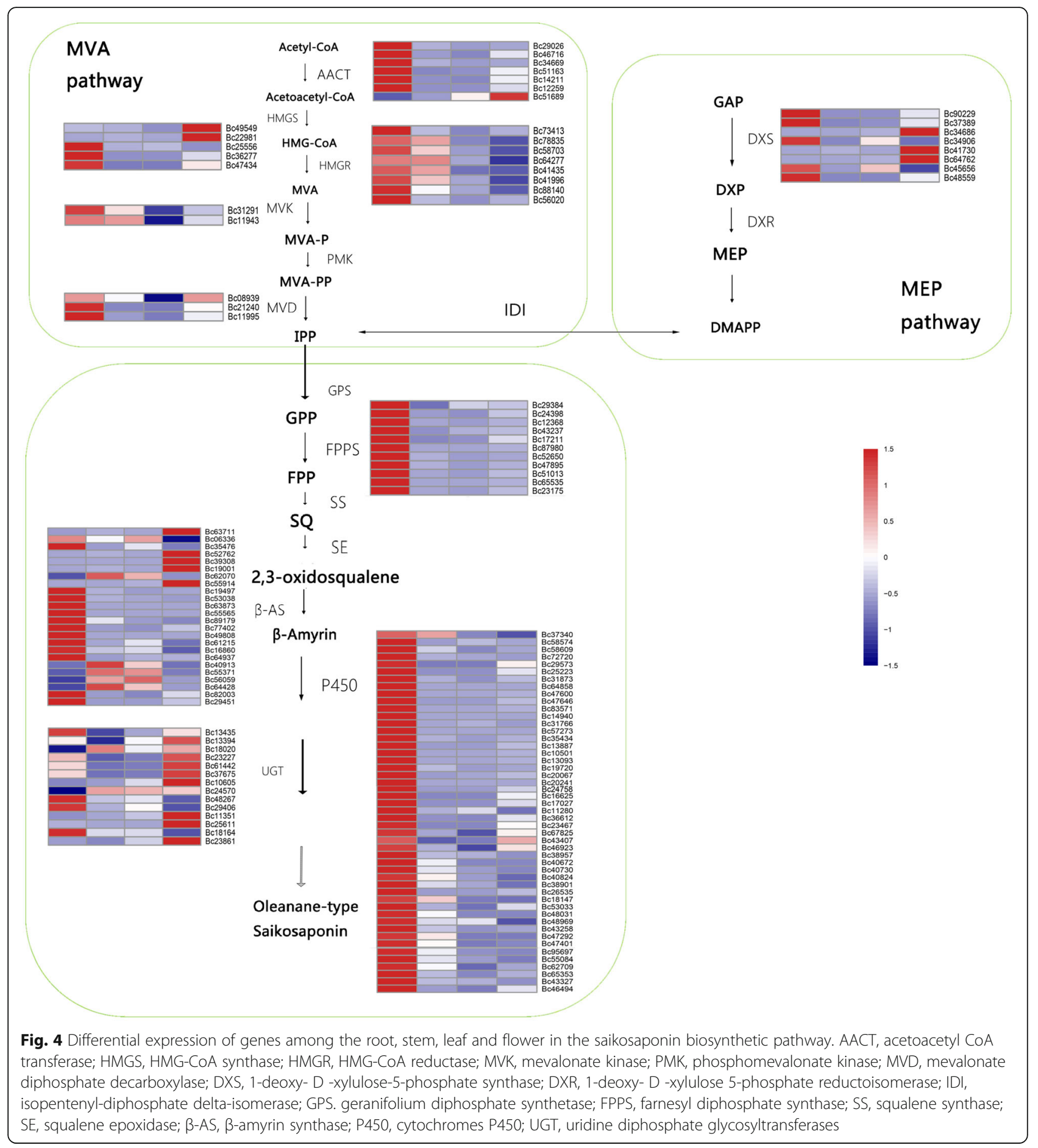

determination of a variety of ginsenosides in Panax ginseng [11]. In the present study, only SSa and SSd were detected in roots by HPLC; we deduced the levels of three SSs types in four organs through a metabolomics method. Therefore, this method could help establish a platform for the detection of secondary metabolites in B. chinense.
To identify the key genes associated with the differences in saikosaponin levels among organs, we analyzed the expression levels of the 131 unigenes based on FPKM values and compared their expression patterns. The results indicated that four proteins encoding acetoacetyl CoA transferase, HMG-CoA synthase, HMG-CoA reductase and mevalonate diphosphate decarboxylase 


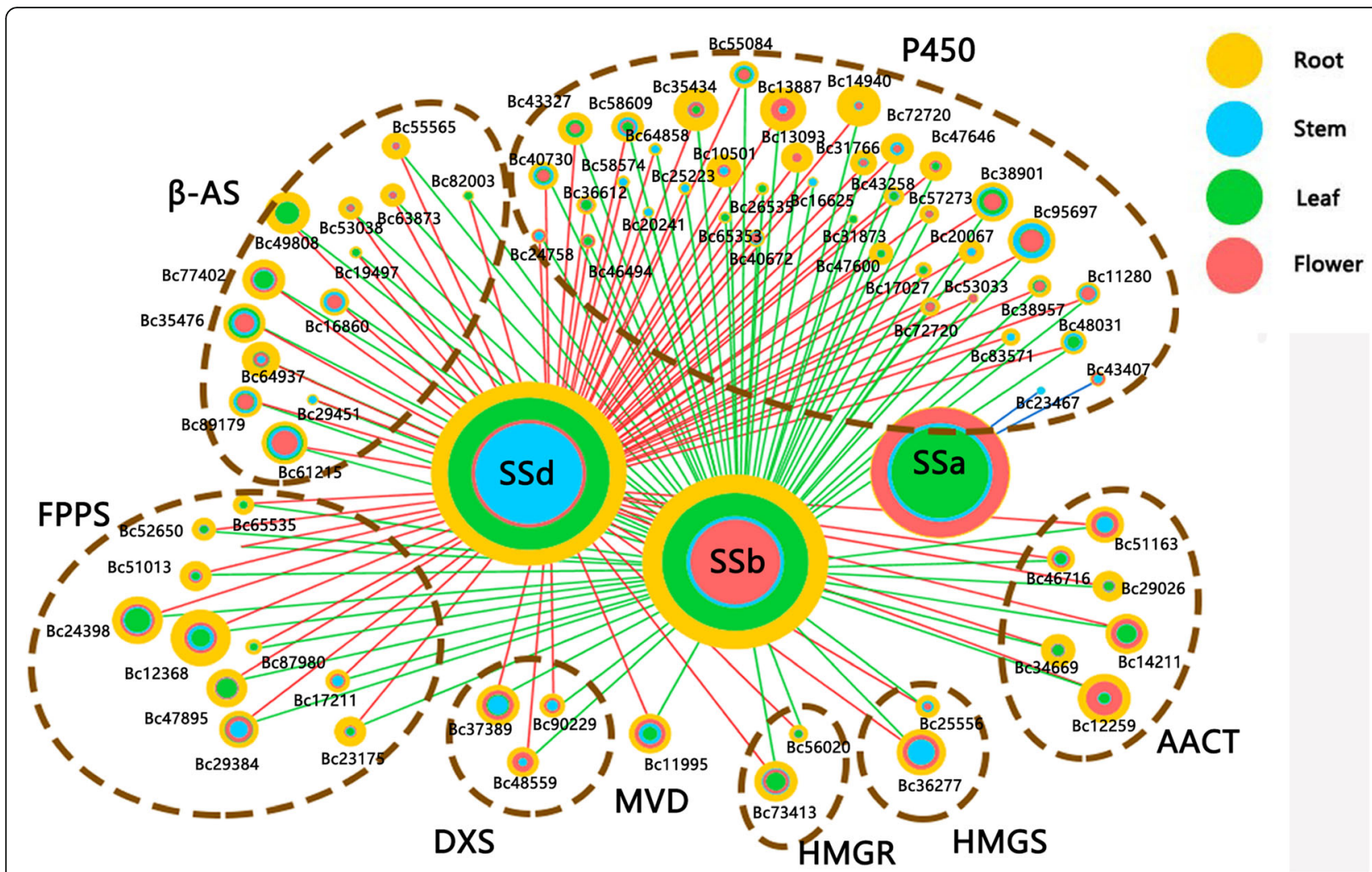

Fig. 5 Connection network between regulatory genes and saikosaponins. AACT, acetoacetyl CoA transferase; HMGS, HMG-CoA synthase; HMGR, HMG-CoA reductase; MVD, mevalonate diphosphate decarboxylase; DXS, 1-deoxy- D -xylulose-5-phosphate synthase; FPPS, farnesyl diphosphate synthase; $\beta$-AS, $\beta$-amyrin synthase; P450, cytochromes P450

were significantly correlated $(P$-value $<0.05)$ with SSd and SSb. In the MVA pathways, AACT is the first enzyme, while HMGR is the first rate-limiting enzyme [15]; therefore, these enzymes might perform important functions in the accumulation of triterpenes. We found that the expression of unigenes Bc12259 (encoding AACT) and Bc73413 (encoding HMGR) in roots was higher than that in other organs. In contrast, except DXS family genes, the unigenes involved in the MEP pathway have no differential expression. Three unigenes (Bc37389, Bc90229, Bc48559) had significant correlations with SSd and SSb. These results are consistent with the findings of other studies showing that the MVA pathway generally supplies precursors for the production of sesquiterpenes and triterpenes and that the MEP pathway generally supplies precursors for the biosynthesis of diterpenoids and carotenoids [16].

With regard to triterpene biosynthetic pathway genes, eleven FPPS unigenes were significantly correlated with SSb and SSd. Among the genes in this family, Bc12368 had the highest expression of roots. In another study on the Bupleurum genus, FPPS expression levels and saikosaponin production increased rapidly when the hairy root of B. falcatum was cultured in 3XRCM [17].
Various studies have explored the functions of FPPS in plant sources [18-20] and confirmed that the synthase catalyzes the reaction of two molecules of IPP with dimethylallyl diphosphate (DMAPP), resulting in geranyl diphosphate (GPP). Besides, all $\beta$-AS unigenes were highly expressed in the root. These results are in accordance with the root of $B$. chinense being the most active organ for terpene biosynthesis. The unigene Bc61215 (encoding $\beta$-AS) was significantly correlated with SSd and SSb. Recently, a genome-wide analysis of Platycodon grandiflorus revealed that the $\beta$-AS gene family played an important role in platycoside biosynthesis, and it had species-specific amplification [21]. In B. kaoi, exogenous application of MeJA increases the expression of $\beta$-AS (by 2-fold) and glycosyltransferase (by 97 -fold), which very likely contributes to increases in SSs [22]. $\beta$-AS has thus been defined as an important branch point between primary and secondary metabolism, and might play a regulatory role in the control of triterpenoid saponin biosynthesis. The cDNAs of a few $\beta$-AS have been cloned, including two different isoforms of $\beta$-AS from B. chinense and one from B. kaoi $[7,23]$.

The hydroxylation and oxidative modification are key factors for immense structural diversity of triterpenoids 

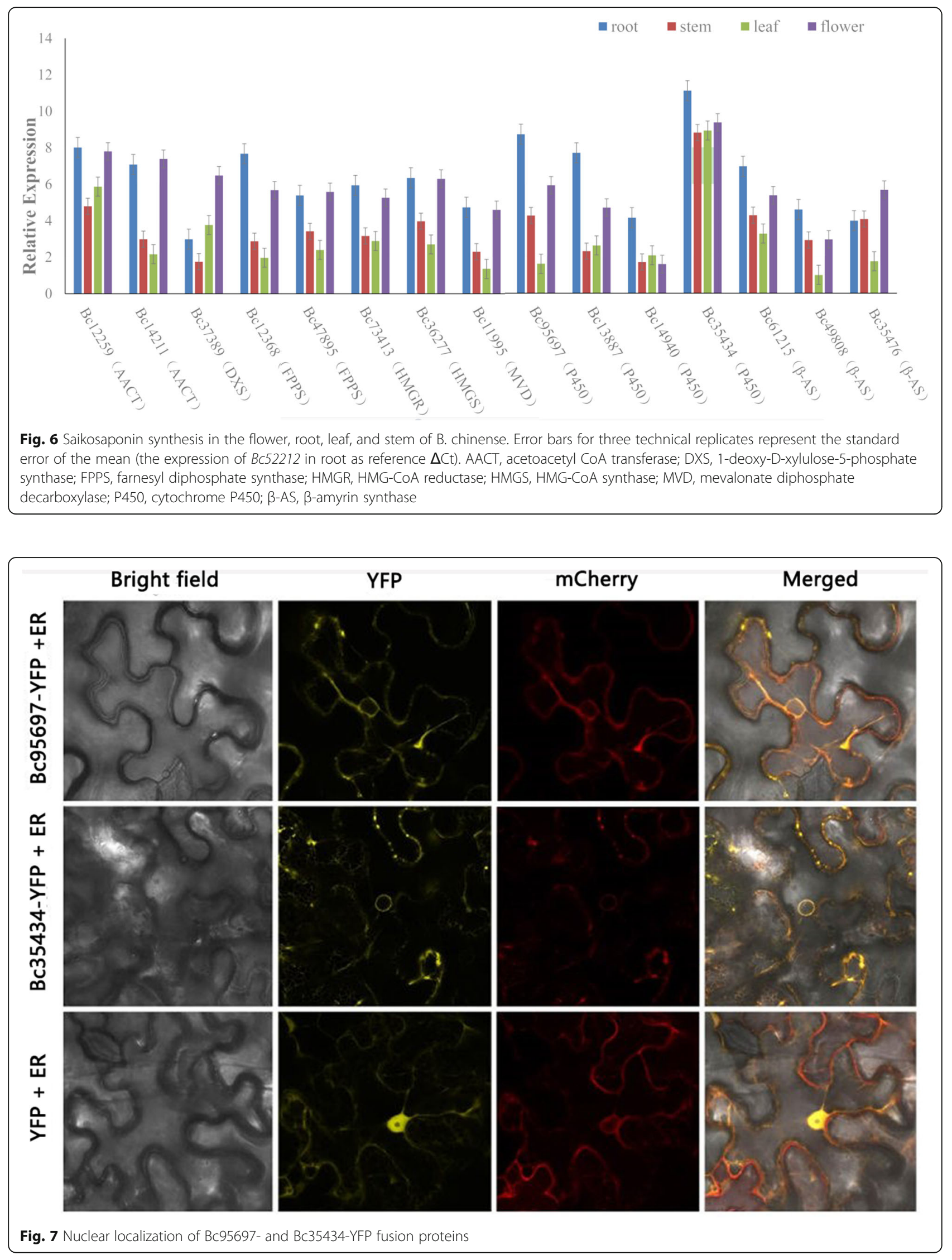


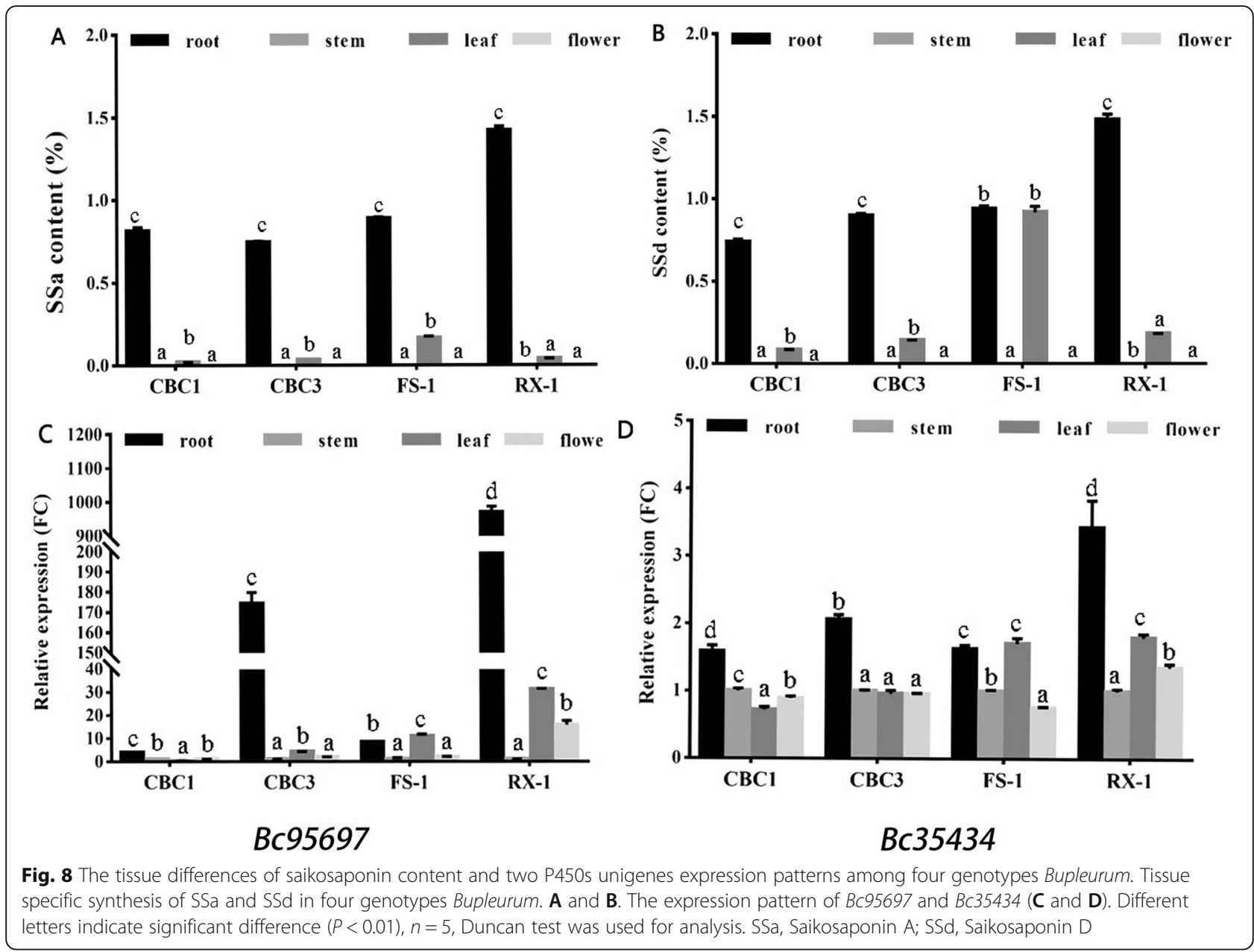

in triterpenoid biosynthesis, and P450s, which mediate oxidations, is the key enzyme. P450s of different P450 gene families, which represent five (CYP 51, 71, 72, 85 and 86) P450 clans, are identified as participating in the synthesis of triterpenoid secondary metabolites [24]. Phylogenetic analysis of more than 400 CYP716s from more than 200 plant and its heterologous expressions in engineering yeasts showed that subgroup A, E, Y, S and C of CYP716 family played an important role in triterpenoid diversity [25]. CYP716Y1, a P450 from B. falcatum was found to catalyzes the $\mathrm{C}-16 \alpha$ hydroxylation of amyrin [2]. In our study, Bc95697 and Bc35434 (encoding P450s) were significantly correlated with $\mathrm{SSb}$ and SSd. Bc95697 belongs to subclass Y, which is highly homologous to CYP716Y1, while Bc35434 belongs to subclass A. Through real-time PCR analysis, the two single genes were highly expressed in plant roots. In addition, the expression levels of these two unigenes in different tissues with multiple genotypes is also highly correlated with the synthesis of saikosaponin. The subcellular localizations of Bc95697 and Bc35434 were localized in the endoplasmic reticulum. Therefore, these two unknown candidate unigenes may be involved in the saikosaponin triterpene biosynthetic pathway.

The materials in the current study were harvested at the adult stage, and four parts were analyzed at the same time: the root, stem, leaf and flower. Compared with the results of studies conducted on plants in the seedling stage, our results can better reflect the synthesis of SSs. This integrated comparison between metabolite contents and the expression of unigenes involved in its biosynthesis demonstrates that biosynthesis of terpenes in $\mathrm{CBC} 1$ is highly controlled by the transcription regulation of the above genes. Furthermore, all the identified ratelimiting enzymes are expected to be useful for various breeding studies on $B$. chinense and possibly other plant species.

\section{Conclusions}

In the current study, we investigated the diversity of the saikosaponin triterpene biosynthetic pathway in the roots, stems, leaves and flowers of $B$. chinense by integrated transcriptomic and metabolomic analysis. Genes from the AACT, FPPS, HMGR and $\beta$-AS families were 
found to play vital roles in regulating saikosaponin biosynthesis, and were related to variations in saikosaponin levels. Bc12259 (encoding AACT), Bc73413 (encoding HMGR), $B c 12368$ (encoding FPPS), $B c 61215$ (encoding $\beta$ AS), Bc95697 and Bc35434 (encoding P450s), which are responsible for the metabolic diversity of these Bupleurum organs, are good candidates to be utilized for the genetic improvement of this important medicinal plant.

\section{Methods}

\section{Plant materials}

The varieties of $B$. chinense genotypes of $\mathrm{CBC} 1, \mathrm{CBC} 3$, FS-1 and RX-1 were applied in current experiment. All of them were bred by Professor Jianhe Wei at the Institute of Medicinal Plant Development (IMPLAD), Chinese Academy of Medical Sciences \& Peking Union Medical College (Beijing, China), and Dr. Ma Yu from Southwest University of Science and Technology (Mianyang, Sichuan, China). CBC1 was the commercial variety of Chuanbeichai No. 1, which originated from northern region of China [26]. CBC3, FS-1, and RX-1 were originated from high rainfall areas of Sichuan province and selected as sources of high sclerotinose resistance. CBC3 was collected from a forest $(800 \mathrm{~m})$ in Wangcang in October 2016. Fs-1 was collected from a medicinal plant farm $(1100 \mathrm{~m})$ in Qingchuan in November 2013. RX-1 was collected from a medicinal plant farm $(600 \mathrm{~m})$ in Rongxian in October 2013. The species of CBC3 was identified by Professor Zechun (Chengdu Institute of Biology, Chinese Academy of sciences, Chengdu, Sichuan, China). FS-1 and RX-1 were identified by Professor Jianhe Wei. The voucher specimens of CBC1 (No.0320120003), CBC3 (No.0320160171), FS-1 (No.0320130129) and RX-1 (No.0320130137) were deposited in the herbarium of School of life science and engineering, Southwest University of Science and Technology, Mianyang, Sichuan, China. All genotypes were planted in a 150 $\mathrm{cm} \times 400 \mathrm{~cm}$ plot at $25 \mathrm{~cm}$ intervals in the greenhouse $\left(14 \mathrm{~h}\right.$ light $/ 10 \mathrm{~h}$ dark at $\left.25^{\circ} \mathrm{C}\right)$ in Mianyang $\left(31^{\circ} 32^{\prime} \mathrm{N}\right.$ and $\left.104^{\circ} 42^{\prime} \mathrm{W}\right)$, Sichuan Province, China. The roots, stems, leaves, and flowers of the plants were collected at the flowering stage in June 2018 and 2020; three biological replications were assessed for each organ. The samples were frozen immediately in liquid nitrogen and kept at $-80^{\circ} \mathrm{C}$ for long-term use.

\section{Metabolite extraction}

Frozen tissues of CBC1 $(100 \mathrm{mg})$ were individually ground with liquid nitrogen, and the ground tissues were resuspended in $500 \mu \mathrm{L}$ of prechilled $80 \%$ methanol (Thermo, United States) and 0.1\% formic acid (Thermo, United States) for vortexing. The samples were incubated on ice for $5 \mathrm{~min}$ and then centrifuged at 15000 rpm in a refrigerated centrifuge (ST16R, Thermo, United States) at $4{ }^{\circ} \mathrm{C}$ for $10 \mathrm{~min}$. Some of the supernatant was diluted to obtain a solution containing 53\% methanol (Thermo, United States) with LC-MS grade water. The samples were subsequently transferred to fresh Eppendorf tubes $(500 \mu \mathrm{L})$ and then centrifuged at $15000 \times g$ and $4{ }^{\circ} \mathrm{C}$ for $20 \mathrm{~min}$. Finally, the supernatants were injected into a HPLC-MS/MS system for analysis. In addition, equal volumes of the different experimental samples were taken and blended as quality control (QC) samples, which were used to evaluate the stability of the results of the whole experimental process and the correlation analysis.

\section{HPLC-MS/MS analysis}

HPLC-MS/MS analyses of CBC1 were performed using an ExionLC ${ }^{\mathrm{mm}}$ AD system (SCIEX, United States) coupled with a QTRAP ${ }^{\circ} 6500+$ mass spectrometer (SCIEX, United States) [27]. The samples were injected onto a BEH C8 column $(2.1 \mathrm{~mm} \times 100 \mathrm{~mm}, 1.9 \mu \mathrm{m}$, Waters, United States) using a 30 min linear gradient at a flow rate of $0.35 \mathrm{~mL} / \mathrm{min}$ in the positive (ESI+) polarity mode. The eluents were eluent A ( $0.1 \%$ formic acid/water) and eluent B ( $0.1 \%$ formic acid/acetonitrile). The solvent gradient was set as follows: mobile phase $B$ was increased linearly from $5 \%$ at $1 \mathrm{~min}$ to $100 \%$ at $24 \mathrm{~min}$ and then held at $100 \%$ until 28.0 min. Finally, solvent B was decreased to $5 \%$ at $28.1 \mathrm{~min}$ and held at $5 \%$ until $30 \mathrm{~min}$. The samples were injected onto an HSS T3 Column $(2.1 \mathrm{~mm} \times 100 \mathrm{~mm}$, Waters, United States) using a 25 min linear gradient at a flow rate of $0.35 \mathrm{~mL} / \mathrm{min}$ for the negative (ESI-) polarity mode. The eluent was the same as that for the ESI+ mode. The solvent gradient was set as follows: mobile phase B was increased linearly from $2 \%$ at $1 \mathrm{~min}$ to $100 \%$ at $18 \mathrm{~min}$ and then held at $100 \%$ until $22.0 \mathrm{~min}$. Finally, solvent B was decreased to $5 \%$ at $22.1 \mathrm{~min}$ and held at $5 \%$ until $25 \mathrm{~min}$.

Mass data acquisition was performed in both ESI+ and ESI- modes using the following parameters: ion spray voltages of $5500 \mathrm{~V}$ in ESI+ mode and $-4500 \mathrm{~V}$ in ESImode, an ion source gas 1 pressure of $55 \mathrm{psi}$, an ion source gas 2 pressure of $55 \mathrm{psi}$, a curtain gas pressure of $30 \mathrm{psi}$, and a turbo spray temperature of $600^{\circ} \mathrm{C}$. The data files generated by HPLC-MS/MS were processed using SCIEX OS Version 1.4 to integrate and correct the peaks. The main parameters were set as follows: minimum peak height, 500; signal/noise ratio, 10; Gaussian smooth width, 3 . The area of each peak represented the relative content of the corresponding substance.

\section{RNA isolation and transcriptome sequencing using the Illumina platform}

Total RNA was extracted from the four organs of $\mathrm{CBC} 1$ using TRIzol (Invitrogen ${ }^{\mathrm{Tm}}$, Life Technologies, Carlsbad, 
United States) according to the manufacturer's protocol. The quality and quantity of the extracted RNA were assessed using an Agilent Bioanalyzer 2000 (Agilent Technologies, Palo Alto, United States). An RNA-Seq library with a 150-bp insertion size was then constructed and sequenced on the Illumina sequencing platform (Novogene, Beijing, China).

\section{Extraction of saikosaponins and HPLC analysis}

For each organ of four genotypes, three biological replications were independently analyzed. In total, 12 samples were randomly analyzed to reduce analysis bias. The SSa and SSd content was determined using a Waters HPLC system (Waters 1525 Binary HPLC Pump, United States) and an Zorbax Extend-C18 column (250 $\mathrm{mm} \times 4.6 \mathrm{~mm}, 5 \mu \mathrm{m}$, Agilent, United States). The reference standards of SSa and SSd were purchased from the National Institutes for Food and Drug Control, Beijing, China. The methods and conditions for determination have been reported previously [28].

\section{Multivariate statistical analysis}

The metabolites were annotated with the KEGG database (https://www.kegg.jp/), the HMDB (http://www. hmdb.ca/) and the LIPID MAPS database (http://www. lipidmaps.org/). The intensities of the mass peaks for each sample were sum-normalized and Pareto-scaled using metaX software [29]. PCA and partial least squares-discrimination analysis (PLS-DA) with data from 12 samples (four organs $\times$ three biological replicates) were performed to observe differences in metabolic composition among the four plant organs. The variable importance in the projection (VIP) values of all metabolites from the PLS-DA were extracted using the first component [30]. We selected metabolites satisfying the following criteria as potential markers: (i) high confidence (VIP value> 1.0) in discriminations between root and stem, between root and leaf, and between root and flower; (ii) mean intensities in one plant organ different from those in another organ $(P$-value $<0.05)$; and (iii) a minimum of a 2-fold change [31]. The $P$-value was calculated using an independent two-sample t-test.

\section{Transcript profiles and annotation}

The sequence data were processed using SMRTlink 5.1 software. Additional nucleotide errors in consensus reads were corrected using the Illumina RNA-Seq, data with the software LoRDEC [32]. Any redundancies in the corrected consensus reads were removed by $\mathrm{CD}$ HIT to obtain the final transcripts for the subsequent analysis [33]. The mapped clean reads for each gene were counted, and the numbers were normalized using the DESeq2 package in $R$ (3.6.2) [34]. Genes showing significantly different expression among the four organs were identified by fold change and binomial tests using the DESeq2 $(\log 2>0 \& P$-value $<0.05)$. The expression levels of genes were determined based on the FPKM values from RNA-Seq data for roots, stems, leaves and flowers using RSEM [35]. The mean FPKM values of the genes were converted to $\log 2$ values, which were used for visualization using the heatmap package in $R$ (3.6.2).

Annotation of the transcriptome was carried out using a BLAST search of nt sequences with an E-value cutoff of $1 \mathrm{e}^{-10}$ and a BLASTx search against the GenBank nr protein [36], Swiss-Prot [37], KEGG [38], KOG [39], Gene Ontology [40], and Pfam (http://pfam.xfam.org/) databases with an E- value cutoff of 1e-10. For each sequence, the top hit was extracted and used for further processing. GO and KEGG pathway functional enrichment analyses were performed by the GOseq $R$ package (3.6.2) and KOBAS software, respectively [41, 42].

\section{Integrative analysis of the metabolome and transcriptome}

Pearson correlation coefficients were calculated for metabolome and transcriptome data integration. For this, the mean content of SSs (SSa, SSb, and SSd) in each organ from the metabolome data and the mean value of the DEGs in each organ from the transcriptome data were used for the correlation analysis. Correlations corresponding to a coefficient with $R^{2}>0.9(P$-value $<0.05)$ were selected (Additional file 1: Table S5). Cytoscape (version 3.6.1) [43] was used to visualize the final interaction network.

\section{Real-time PCR analysis}

Fifteen genes potentially involved in the saikosaponin synthesis of $B$. chinense were selected to perform expression pattern analysis. Total RNA was extracted by TRIzol (Invitrogen ${ }^{\mathrm{Tw}}$, Life Technologies, Carlsbad, United States) from independent biological samples collected at the same stage as the ones used for RNA-Seq analysis and using as described above. The cDNAs were synthesized by the TransScript All-in-One First-Strand cDNA Synthesis SuperMix kit (TransStart, Beijing, China) using specific primer pairs [44] (Additional file 1: Table S6). Real-time PCR was performed using CFX96 Touch RealTime PCR Detection System (Bio-Rad, Hercules, CA, USA) with Green qPCR SuperMix UDG kit (TransStart, Beijing, China). The PCR reactions were conducted as follows: $30 \mathrm{~s}$ at $95^{\circ} \mathrm{C}$ for denaturation, $5 \mathrm{~s}$ at $94^{\circ} \mathrm{C}, 15 \mathrm{~s}$ at $60^{\circ} \mathrm{C}$ for annealing, and $10 \mathrm{~s}$ at $72{ }^{\circ} \mathrm{C}$ for extension. The relative abundance of transcripts was calculated using the $2^{-\Delta \Delta \mathrm{Ct}}$ formula [45]. In the assays, the $\beta$ action gene $B c A D F 7$ was used as the housekeeping gene for normalization. The experiments were performed for three technical replications. 


\section{Subcellular localization}

To further understand the biological roles of P450s enzymes, we conducted the intracellular localization of P450s expressed with YFP fusions transiently in Nicotiana benthamiana leaves by confocal microscopy. The fusion expression vector of $B c 95697$ and $B c 35434$ genes were constructed using specific primer pairs (Additional file 1: Table S6). The two DNA segments were inserted into the pC131-YFP vector to generate Bc95697-YFP and Bc35434-YFP fusion proteins. The two recombinant vectors were transferred into Agrobacterium tumefaciens GV3101 strains and suspended in the mixture solution with $10 \mathrm{mM} \mathrm{MgCl} 2,10 \mathrm{mM} \mathrm{MES}$, and $100 \mu \mathrm{M}$ acetosyringone (Sigma-Aldrich), $\mathrm{pH}$ 5.6. After incubation for $3 \mathrm{~h}$ at room temperature, the strain was transfused into leaves of $N$. benthamiana plants. Fluorescence images of the YFP and mCherry signals were visualized using a laser scanning confocal microscope (Leica TCS SMD FCS, Germany) after 3 days. The excitation and wavelength emissions were 514 and $530-580 \mathrm{~nm}$ for YFP, $587 \mathrm{~nm}$ and $610-650 \mathrm{~nm}$ for mCherry, respectively.

\section{Abbreviations}

IPP: Isopentenyl diphosphate; DMAPP: Dimethylallyl diphosphate; MVA: Mevalonate; MEP: Methylerythritol phosphate; AACT: Acetoacetyl CoA transferase; HMGS: HMG-CoA synthase; HMGR: HMG-CoA reductase; MVK: Mevalonate kinase; PMK: Phosphomevalonate kinase; MVD: Mevalonate diphosphate decarboxylase; DXS: 1-deoxy- D -xylulose-5-phosphate synthase; DXR: 1-deoxy- D -xylulose 5-phosphate reductoisomerase; IDI: Isopentenyldiphosphate delta-isomerase; GPS: Geranyl pyrophosphate synthetase; FPPS: Farnesyl diphosphate synthase; SS: Squalene synthetase; SE: Squalene epoxidase; $\beta$-AS: $\beta$-amyrin synthase; P450s: Cytochrome P450 enzymes; UGTs: Uridine diphosphate glycosyltransferases; SSs: Saikosaponins; SSa: Saikosaponin A; SSd: Saikosaponin D; SSb: Saikosaponin B; nr: Nonredundant; nt: Nucleotide; GO: Gene ontology; KEGG: Kyoto Encyclopedia of Genes and Genomes; DEGs: Differentially expressed genes; HPLC-MS/ MS: High-performance liquid chromatography-tandem mass spectrometry; QC: Quality control; RNA-seq: RNA sequencing; PCA: Principal component analysis; PCR: Polymerase chain reaction; YFP: Yellow fluorescent protein; LCMS: Liquid chromatography-mass spectrometry; PLS-DA: Partial least squaresdiscrimination analysis; VIP: Variable importance in the projection

\section{Supplementary Information}

The online version contains supplementary material available at https://doi. org/10.1186/s12864-021-08144-6.

Additional file 1: Table S1. A list of 600 metabolites identified in four organs. Table S2. Mean content of Saikosaponin A (SSa) and Saikosaponin D (SSd) among the root, stem, leaf, flower. Table S3. Summary of unigene ID and sequence of B.Chinese. Table S4. FPKM values of unigenes involved in the saikosaponin synthesis pathways among the root, stem, leaf and flower. Table S5. Interaction value between metabolite and gene. Table S6. Primer list used in real-time PCR analysis.

\section{Acknowledgements}

The authors would like to acknowledge and thank Prof. Zheyong Xue from Northeast Forestry University for paper editing.

\section{Authors' contributions}

YLH wrote the manuscript. HC performed the data analysis. YXY and JZ performed the transcriptome analysis. BY performed the metabolome analysis. LF seeded Chuanbeichai No.1. YGZ and PW performed the HPLC analysis. DBH and MY bred out the commercial varieties of Chuanbeichai No.1. MY bred out the genotypes of CBC3, FS-1 and RX-1. JNZ and MY funded the whole project and helped YLH to complete the manuscript. All authors have read and approved the manuscript.

\section{Funding}

This work was supported by National Natural Science Foundation of China (81603223), China Agriculture Research System of MOF and MARA, Young Elite Scientists Sponsorship Program by CACM (2019-QNRC2-C15), CAMS Innovation Fund for Medical Sciences (CIFMS) under grant number 201612M-2-003, the Programs of Science and Technology Department of Sichuan Province (2019YFH0072), the Doctoral Research Funding of Southwest University of Science and Technology (19zx7117 and 21zx7116).

\section{Availability of data and materials}

The raw sequence data generated in this study have been deposited in the Short Read Archive (SRA) of the NCBI under accession number PRJNA728560 (https://dataview.ncbi.nlm.nih.gov/object/PRJNA728560).

\section{Declarations}

\section{Ethics approval and consent to participate}

All the samples from the Bupleurum L. were cultivated varities and collected from the natural product research centre of Southwest University of Science and Technology. Collection of plant materials complied with the institutional, national and international guidelines. No specific permits were required. Vouchers of this species have been placed in the Southwest University of Science and Technology.

Consent for publication

Not applicable.

\section{Competing interests}

The authors declare that they have no competing interests.

\section{Author details}

${ }^{1}$ School of life science and engineering, Southwest University of Science and Technology, 59 Qinglong Road, Mianyang 621010, Sichuan, China. ${ }^{2}$ Institute of Medicinal Plant Resources, Sichuan Academy of Traditional Chinese Medicine Sciences, 51 4th Section S. Renmin Road, Chengdu 610041, Sichuan, China. ${ }^{3}$ Sichuan Institute for Translational Chinese Medicine, Chengdu 610041, China. ${ }^{4}$ Laboratory of Medicinal Plant Cultivation, Institute of Medicinal Plant Development (IMPLAD), Chinese Academy of Medical Sciences \& Peking Union Medical College, Beijing 100193, China.

Received: 26 May 2021 Accepted: 25 October 2021

Published online: 19 November 2021

References

1. Ashour ML, Wink M. Genus Bupleurum: A review of its phytochemistry, pharmacology and modes of action. J Pharm Pharmacol. 2011;63:305-21.

2. Moses T, Pollier J, Almagro L, Buyst D, Van-Montagu M, Pedreno MA, et al. Combinatorial biosynthesis of sapogenins and saponins in Saccharomyces cerevisiae using a C-16 alpha hydroxylase from Bupleurum falcatum. Proc Natl Acad Sci. 2014;111:1634-9.

3. Jiang H, Yang L, Hou A, Zhang J, Wang S, Man W, et al. Botany, traditional uses, phytochemistry, analytical methods, processing, pharmacology and pharmacokinetics of Bupleuri Radix, A systematic review. Biomed Pharmacother. 2020;131:110679.

4. Hirai MY, Yano M, Goodenowe DB, Kanaya S, Kimura T, Awazuhara M, et al. Integration of transcriptomics and metabolomics for understanding of global responses to nutritional stresses in Arabidopsis thaliana. Proc Natl Acad Sci U S A. 2004;101:10205-10.

5. Lin TY, Chiou CY, Chiou SJ. Putative genes involved in saikosaponin biosynthesis in Bupleurum species. Int J Mol Sci. 2013;14:12806-26.

6. Zheng X, Xu H, Ma X, Zhan R, Chen W. Triterpenoid Saponin biosynthetic pathway profiling and candidate Gene Mining of the llex asprella root using RNA-Seq. Int J Mol Sci. 2014;15:5970-87.

7. Sui C, Zhang J, Wei J, Chen S, Li Y, Xu J, et al. Transcriptome analysis of Bupleurum chinense focusing on genes involved in the biosynthesis of saikosaponins. BMC Genomics. 2011;12:1-16. 
8. Mounet F, Moing A, Garcia V, Petit J, Maucourt M, Deborde C, et al. Gene and Metabolite regulatory network analysis of early developing fruit tissues highlights new candidate genes for the control of tomato fruit composition and development. Plant Physiol. 2009;149:1505-28.

9. Yonekura-Sakakibara K, Tohge T, Matsuda F, Nakabayashi R, Takayama H, Niida R, et al. Comprehensive flavonol profiling and transcriptome coexpression analysis leading to decoding gene-metabolite correlations in Arabidopsis. Plant Cell. 2008;20:2160-76.

10. Cho K, Cho KS, Sohn HB, Ha IJ, Hong SY, Lee H, et al. Network analysis of the metabolome and transcriptome reveals novel regulation of potato pigmentation. J Exp Bot. 2016;67:1519-33.

11. Lee YS, Park HS, Lee DK, Jayakodi M, Kim NH, Koo HJ, et al. Integrated transcriptomic and Metabolomic analysis of five Panax ginseng cultivars reveals the dynamics of Ginsenoside biosynthesis. Front Plant Sci. 2017; 8:1048.

12. Pan SL. Bupleurum species: scientific evaluation and clinical applications: CRC Press; 2006

13. Huang HQ, Zhang $X$, Xu ZX, Su J, Yan SK, Zhang WD. Fast determination of saikosaponins in Bupleurum by rapid resolution liquid chromatography with evaporative light scattering detection. J Pharm Biomed Anal. 2009;49:1048-55.

14. Sumner LW, Lei Z, Nikolau BJ, Saito K. Modern plant metabolomics: advanced natural product gene discoveries, improved technologies, and future prospects. Nat Prod Rep. 2015;32:212-29.

15. Tan Y, Tang CH, Feng J, Feng N, Wu YY, Bao DP, et al. Current Progress in the study on biosynthesis and regulation of Ganoderma triterpenoids. Acta Edulis Fungi. 2019;26:125-40.

16. Rodriguez-Concepcion M, Boronat A. Elucidation of the methylerythritol phosphate pathway for isoprenoid biosynthesis in bacteria and plastids. A metabolic milestone achieved through genomics. Plant Physiol. 2002;130: 1079-89.

17. Kim YS, Cho JH, Ahn JC, Hwang B. Upregulation of isoprenoid pathway genes during enhanced saikosaponin biosynthesis in the hairy roots of Bupleurum falcatum. Mol Cells (Springer Science \& Business Media BV). 2006; 22:269-74.

18. Yu Z, Yang L, Han M, Yang L. Correlation between content of saikosaponin and expression of key enzyme genes in different parts of Bupleurum chinense. Chin Tradit Herbal Drugs. 2019;50:2433-41.

19. Ding $Y X$, Xiang OY, Chang $C H$, Ren A, Shi L, Li YX, et al. Molecular cloning, characterization, and differential expression of a farnesyl-diphosphate synthase gene from the basidiomycetous fungus Ganoderma lucidum. Biosci Biotechnol Biochem. 2008;72:1571-9.

20. Cunillera N, Arro M, Delourme D, Karst F, Boronat A, Ferrer A. Arabidopsis thaliana contains two differentially expressed farnesyl-diphosphate synthase genes. J Biol Chem. 1996;72:1571-9.

21. Kim J, Kang SH, Park SG, Yang TJ, Lee Y, Kim OT, et al. Whole-genome, transcriptome, and methylome analyses provide insights into the evolution of platycoside biosynthesis in Platycodon grandiflorus, a medicinal plant. Horticulture Res. 2020;7:1-12.

22. Chen LR, Chen YJ, Lee CY, Lin TY. MeJA-induced transcriptional changes in adventitious roots of Bupleurum kaoi. Plant Sci. 2007;173:12-24.

23. Lin WY, Peng PH, Lin TY. Cloning and characterization of beta-amyrin synthase from Bupleurum kaoi. In: 8th international congress of plant molecular biology. Book of abstracts, ISPMB; 2006.

24. Sawai S, Saito K. Triterpenoid biosynthesis and engineering in plants. Front Plant Sci. 2011;2:25.

25. Miettinen K, Pollier J, Buyst D, Arendt P, Csuk R, Sommerwerk S, et al. The ancient CYP716 family is a major contributor to the diversification of eudicot triterpenoid biosynthesis. Nat Commun. 2017;8:1-13.

26. Yu M, Chen H, Liu SH, Li YC, Sui C, Hou DB, et al. Differential expression of genes involved in Saikosaponin biosynthesis between Bupleurum chinense DC. And Bupleurum scorzonerifolium Willd. Front Genet. 2020;11:583245.

27. Dunn WB, Broadhurst D, Begley P, Zelena E, Francis-Mclntyre S, Anderson N, et al. Procedures for large-scale metabolic profiling of serum and plasma using gas chromatography and liquid chromatography coupled to mass spectrometry. Nat Protoc. 2011;6:1060-83.

28. Xu J, Wu SR, Xu YH, Ge ZY, Sui C, Wei JH. Overexpression of BcbZIP134 negatively regulates the biosynthesis of saikosaponins. Plant Cell Tissue Organ Culture (PCTOC). 2019;137:297-308.

29. Wen B, Mei Z, Zeng C, Liu S. metaX: a flexible and comprehensive software for processing metabolomics data. BMC Bioinformatics. 2017;18:1-14.
30. Kieffer DA, Piccolo BD, Vaziri ND, Liu S, Lau WL, Khazaeli M, et al. Resistant starch alters gut microbiome and metabolomic profiles concurrent with amelioration of chronic kidney disease in rats. Am J Physiol Ren Physiol. 2016:310:F857-71.

31. Heischmann S, Quinn K, Cruickshank-Quinn C, Liang LP, Reisdorph R, Reisdorph N, et al. Exploratory metabolomics profiling in the kainic acid rat model reveals depletion of 25-hydroxyvitamin D3 during epileptogenesis. Sci Rep. 2016;6:1-14.

32. Salmela L, Rivals E. LoRDEC: accurate and efficient long read error correction. Bioinformatics. 2014;30:3506-14.

33. Fu L, Niu B, Zhu Z, Wu S, Li W. CD-HIT: accelerated for clustering the nextgeneration sequencing data. Bioinformatics. 2012;28:3150-2.

34. Anders S. Analysing RNA-Seq data with the DESeq package. Mol Biol. 2010; $43: 1-17$.

35. Li B, Dewey CN. RSEM: accurate transcript quantification from RNA-Seq data with or without a reference genome. BMC Bioinformatics. 2011;12:1-16.

36. Li W, Jaroszewski L, Godzik A. Tolerating some redundancy significantly speeds up clustering of large protein databases. Bioinformatics. 2002;18:77-82.

37. Bairoch A, Apweiler R. The SWISS-PROT protein sequence database and its supplement TrEMBL in 2000. Nucleic Acids Res. 2000;28:45-8.

38. Kanehisa M, Goto S, Kawashima S, Okuno Y, Hattori M. The KEGG resource for deciphering the genome. Nucleic Acids Res. 2004;32(suppl 1):D277-80.

39. Tatusov RL, Fedorova ND, Jackson JD, Jacobs AR, Kiryutin B, Koonin EV, et al. The COG database: an updated version includes eukaryotes. BMC Bioinformatics. 2003;4:1-14.

40. Ashburner M, Ball CA, Blake JA, Botstein D, Butler H, Cherry JM, et al. Gene ontology: tool for the unification of biology. Nat Genet. 2000;25:25-9.

41. Young MD, Wakefield MJ, Smyth GK, Oshlack A. Gene ontology analysis for RNA-seq: accounting for selection bias. Genome Biol. 2010;11:R14.

42. Bu DC, Luo HT, Huo PP, Wang ZH, Zhang S, He ZH, et al. KOBAS-i: intelligent prioritization and exploratory visualization of biological functions for gene enrichment analysis. Nucleic Acids Res. 2021;49:W317-25.

43. Shannon P, Markiel A, Ozier O, Baliga NS, Wang JT, Ramage D, et al. Cytoscape: a software environment for integrated models of biomolecular interaction networks. Genome Res. 2003;13:2498-504.

44. Lalitha S. Primer premier 5. Biotech Software Internet Report: The Computer Software Journal for Scient. 2000;1:270-2.

45. Bao ZZ, Zhang KD, Lin HF, Li CJ, Zhao XR, Wu J, et al. Identification and selection of reference genes for quantitative transcript analysis in Corydalis yanhusuo. Genes. 2020;11:130.

\section{Publisher's Note}

Springer Nature remains neutral with regard to jurisdictional claims in published maps and institutional affiliations.

Ready to submit your research? Choose BMC and benefit from:

- fast, convenient online submission

- thorough peer review by experienced researchers in your field

- rapid publication on acceptance

- support for research data, including large and complex data types

- gold Open Access which fosters wider collaboration and increased citations

- maximum visibility for your research: over $100 \mathrm{M}$ website views per year

At BMC, research is always in progress.

Learn more biomedcentral.com/submissions 